\title{
New Technology for Fabric Defect Detection Based on K-means Algorithm
}

\author{
Sheetal Thorave ${ }^{1}$, Prof.M.S.Biradar ${ }^{2}$ \\ ${ }^{1}$ (Student, Department of EXTC, Siddhant College of Engineering, Pune, India) \\ ${ }_{2}^{2}$ (Professor, Department, of EXTC, Siddhant College of Engineering, Pune, India)
}

\begin{abstract}
There are various existing computer-based researches available in fabric defect detection but various problems like inaccuracy exists in these techniques. So in this paper, we have introduced the new technique for fabric defect detection using automatic visual analysis. In this paper we used the clustering algorithm for fabric defect detection. Clustering methods is also used for image segmentation. The main focus of this paper is to use the K-means clustering technique to achieve highly accurate and reliable fabric defect detection within reasonable computing time.
\end{abstract}

Keywords: - Fabric defect detection, Preprocessing, Clustering, K-mean algorithm, Thersholding.

\section{INTRODUCTION}

The fabric defect detection is an important issue in recent year. There are many computer based technology is available in defect detection so in this paper we introduced image reorganization techniques are being increasingly used to automate the detection of fabric defects in recent years. The approaches of fabric feature extraction have been mainly classified into various categories. In this paper, we introduced algorithms $\mathrm{K}$-means algorithm. The k-mean method is based on clustering .Clustering techniques are unsupervised learning methods of grouping similar from dissimilar data types. Therefore, these are popular for various data mining and pattern recognition purposes. Segmentation is the process of partitioning a digital image into multiple segments. The goal of segmentation is to simplify and/or change the representation of an image into something that is more meaningful easier to analyze. Clustering is one of the methods used for segmentation.

Cluster analysis is also recognized as an important technique for classifying data, finding clusters of a dataset based on similarities in the same cluster and dissimilarities between different clusters [7][9]. Putting each point of the dataset to exactly one cluster is the basic of the conventional clustering method where as clustering algorithm actually partitions unlabeled set of data into different groups according to the similarities. As such there are many algorithms that are proposed to improve the clustering performance. Clustering is basically considered as classification of similar objects or in other words, it is precisely partitioning of datasets into clusters so that data in each cluster shares some common trait.

In this research paper, we recognize the fabric defect detection using the K-Means clustering algorithm are analyzed based on their clustering efficiency.

\section{PROPOSED METHODS}

The below block diagram is proposed method to fabric defect detection. It uses the combination of two algorithms for image segmentation. The proposed method consists of main five modules. Each of module and its function will be explained below in details.

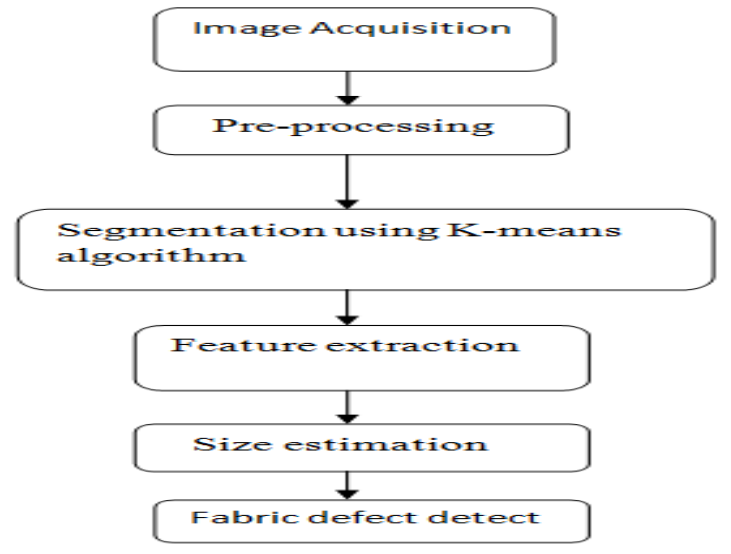

Fig.1. block diagram of proposed method. 


\section{PRE-PROCESSING}

Pre-processing is applied on images at the lowest level of abstraction and its aim is to reduce undesired distortions and enhance the image data which is useful and important for further processing [1]. It is usually necessary and required for improving the performance of image processing methods like image transform, segmentation, feature extraction and fault detection [2] [3]. This paper is focused on filtering and intensity adjustment as pre-processing methods. According to the need of the next level is the pre processing step which convert the image. It performs filtering of noise and other artifacts in the image and sharpening the edges in the image. RGB to grey conversion and Reshaping also takes place in pre-processing. It also includes median filter for noise removal. It may arrive due to the thermal effect. The main aim of this paper is to detect the Defect in fabric. But for the complete system it needs the process of noise removal. For better understanding the function of median filter, we added the salt and pepper noise artificially and removing it using median filter.

\subsection{Conversion RGB to Gray:}

A grayscale digital image is an image in which the value of each pixel is a single sample, that is, it carries only intensity information [4]. Images of this sort, also known as black-and-white, are composed exclusively of shades of gray, varying from black at the weakest intensity to white at the strongest. Grayscale images are distinct from one-bit bi-tonal black-and-white images, which in the context of computer imaging are images with only the two colors, black, and white (also called bit level or binary images). Grayscale images have many shades of gray in between.

\subsection{Median filter:}

In image processing, it is often desirable to be able to perform some kind of noise reduction on an image. The median filter is a nonlinear digital filtering technique, often used to remove noise[5][6]. Such noise reduction is a typical pre-processing step to improve the results of later processing (for example, edge detection on an image). Median filtering is very widely used in digital image processing because, under certain conditions, it preserves edges while removing noise.

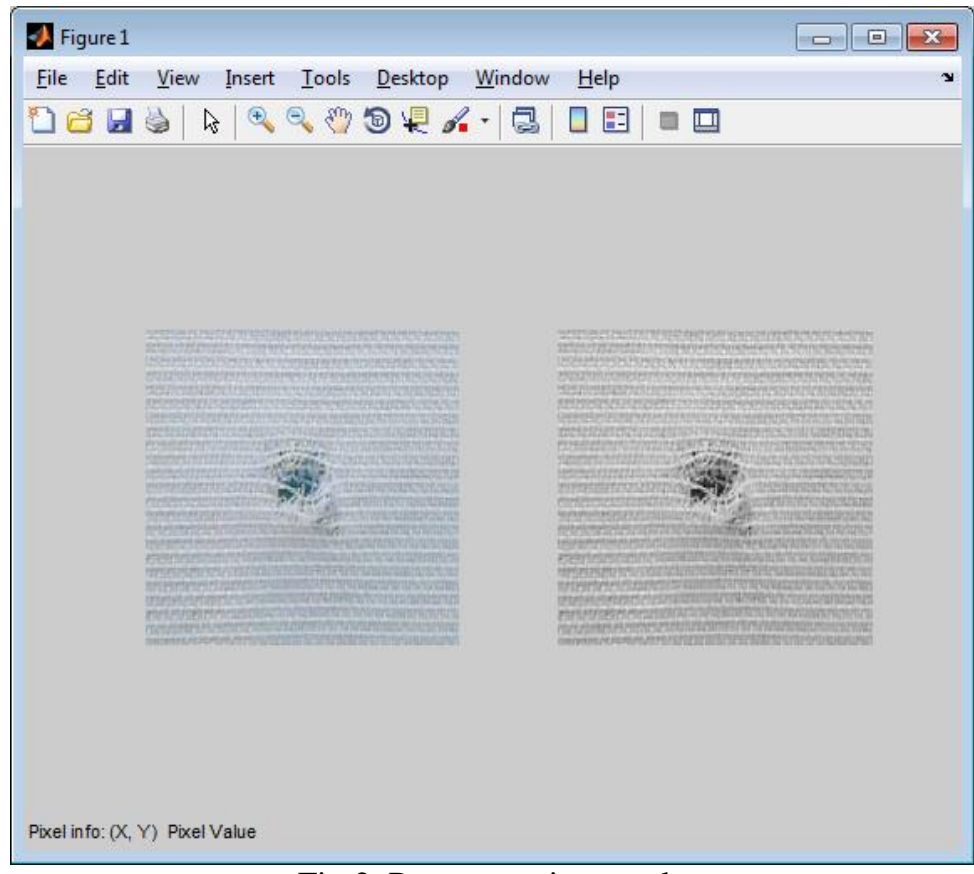

Fig.2 Pre-processing result.

\section{K-MEANS SEGMENTATION}

\subsection{K-means clustering:}

$\mathrm{K}$-means is one of the simplest unsupervised learning algorithms that solve the well known clustering problem.[7][8][10] The procedure follows a simple and easy way to classify a given data set through a certain number of clusters (assume $\mathrm{k}$ clusters) fixed a priori. The main idea is to define $\mathrm{k}$ centroids, one for each cluster. These centroids should be placed in a cunning way because of different location causes different result. So, the better choice is to place them as much as possible far away from each other. The next step is to take each point belonging to a given data set and associate it to the nearest centroid. When no point is pending, the first step is completed and an early group age is done. At this point we need to re-calculate $\mathrm{k}$ new centroids as bar centers of 
the clusters resulting from the previous step. After we have these k new centroids, a new binding has to be done between the same data set points and the nearest new centroid. A loop has been generated. As a result of this loop we may notice that the $\mathrm{k}$ centroids change their location step by step until no more changes are done. In other words centroids do not move any more.

4.2 Mathematical Equation:

For a given image, compute the cluster means $\mathrm{m}$

$M=\frac{\sum_{j x(i)=k} x_{i}}{N_{k}}, k 1, \ldots K-(1)$

Calculate the distance between the cluster center to each pixel

$D(i)=\operatorname{argmin}\left\|x_{i}-M_{k}\right\|^{2}, i=1, \ldots . . N-$ (2)

Repeat the above two steps until mean value convergence.

4.3. Flowchart of k-means algorithm:

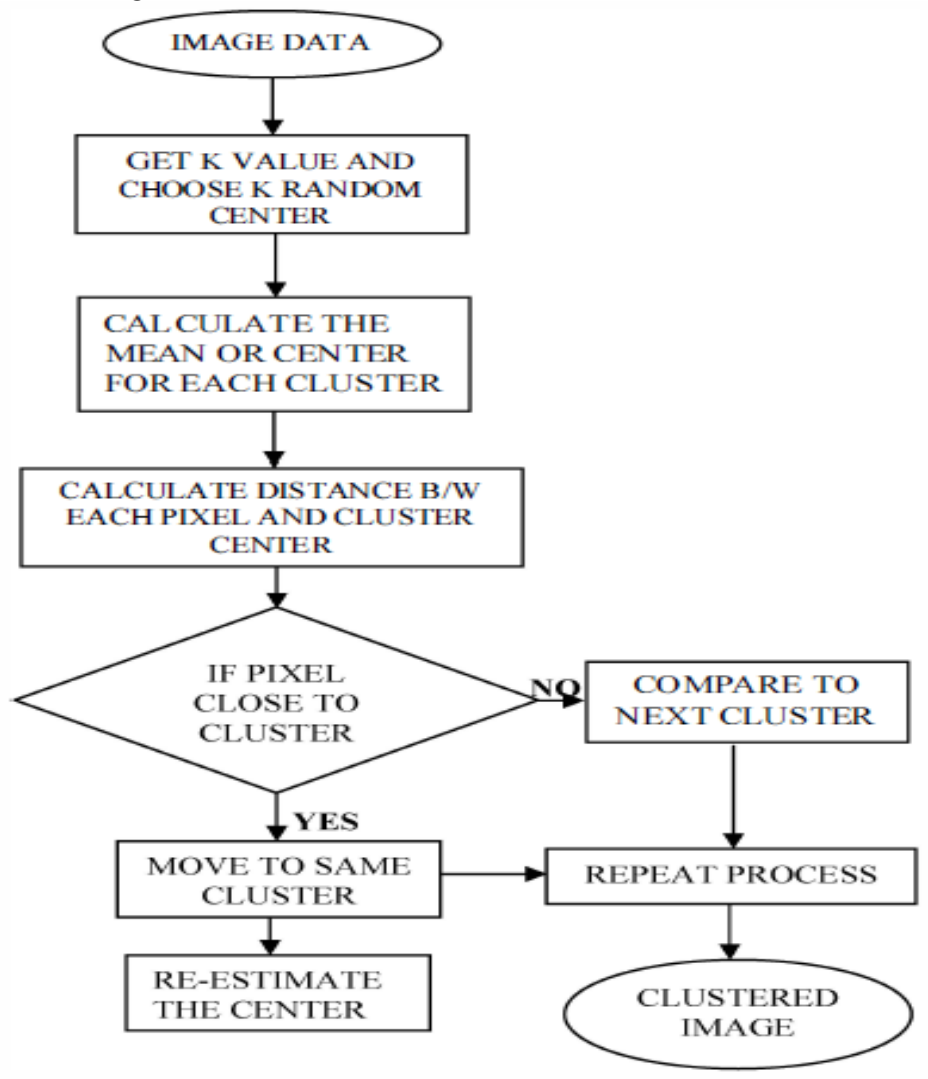

Fig.3 Representation of the k-means algorithm and its flow.

\subsection{Algorithm:}

1. Give the number of cluster value as $\mathrm{k}$.

2. Then you have to randomly choose the k cluster centers.

3. Calculate mean or center of all the cluster.

4. Calculate the distance b/w each pixel to each cluster center

5. If the distance is near to the center then move to that cluster.

6. Otherwise move to next cluster.

7. Re-estimate the center.

8. Repeat the process until the center doesn't move. 


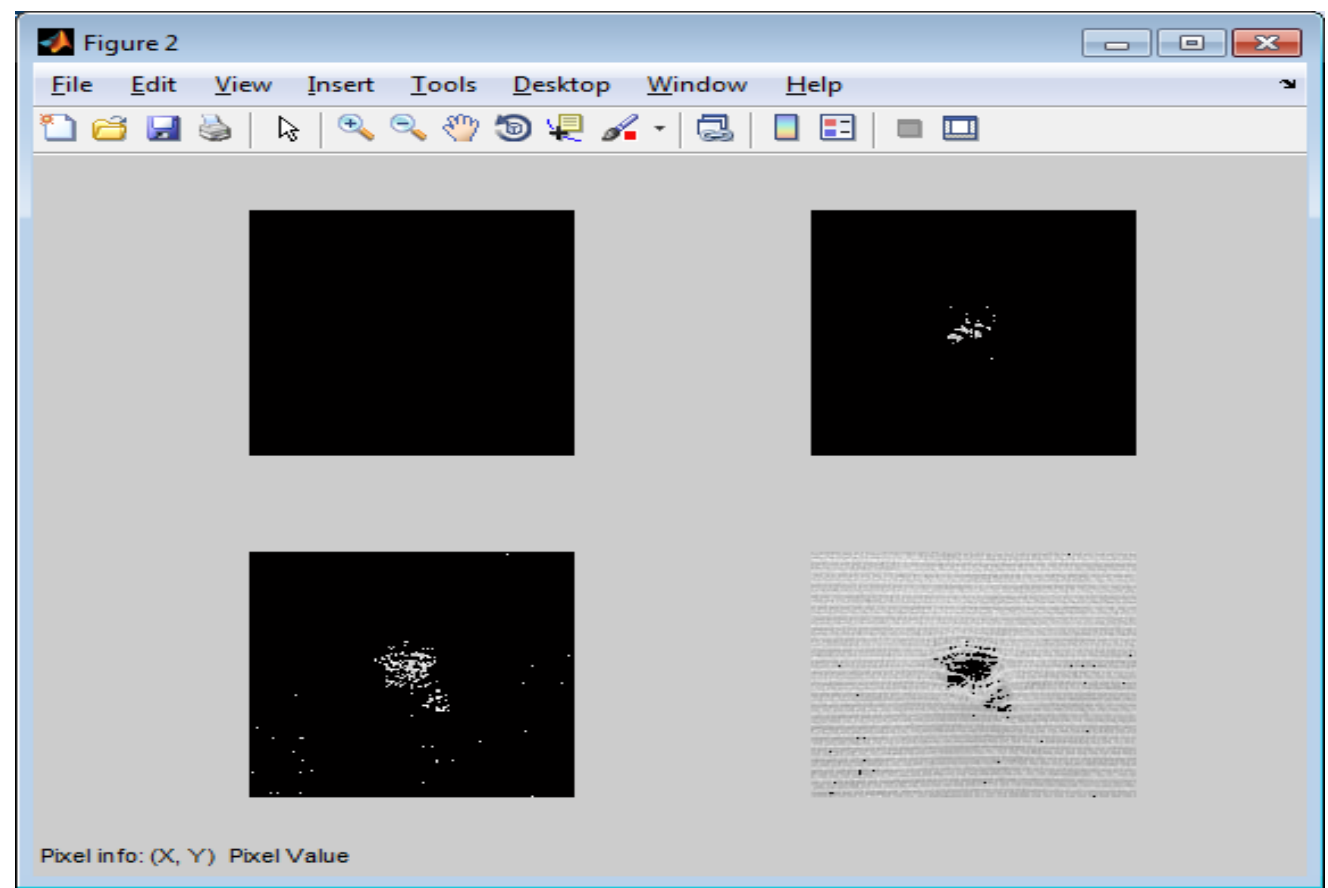

Fig.4 K-means result.

\section{EXAMPLE DEMONSTRATION}

Thus K-means, which is a greedy algorithm, can only converge to a local minimum, even though recent study has shown with a large probability K-means could converge to the global optimum when clusters are well separated (Meila, 2006) [9]. K-means starts with an initial partition with $\mathrm{K}$ clusters and assign patterns to clusters so as to reduce the squared error. Since the squared error always decreases with an increase in the number of clusters $\mathrm{K}$ ( with $\mathrm{J}(\mathrm{C})=0$ when $\mathrm{K}=\mathrm{n}$ ), it can be minimized only for a fixed number of clusters. The main steps of K-means algorithm are as above(Jain and Dubes, 1988) Fig. 3 shows an illustration of the Kmeans algorithm on a 2-dimensional dataset with three clusters first upon we Select an initial partition with $\mathrm{K}$ clusters; repeat steps 2 and 3 until cluster membership stabilizes. Generate a new partition by assigning each pattern to its closest cluster center and then Compute new cluster centers.

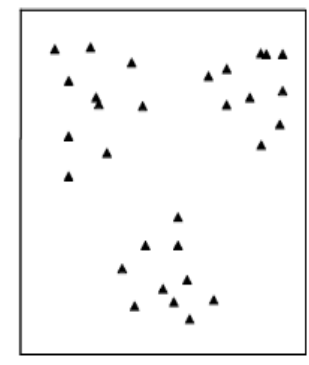

(a) Input data

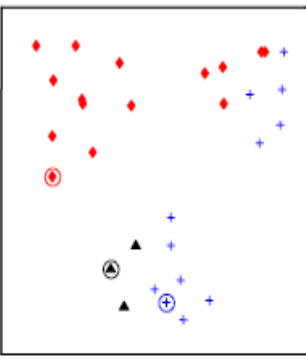

(b) Seed point selection

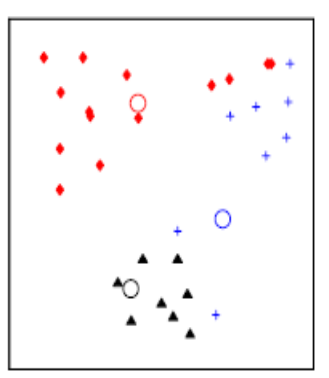

(c) Iteration 2

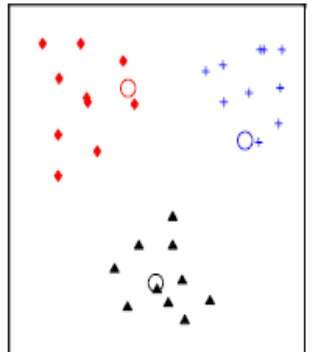

(d) Iteration 3

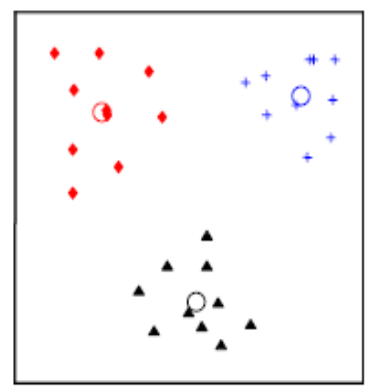

(e) Final clustering

Fig.3 Illustration of K-means algorithm 
In this figure shows (a) Two-dimensional input data with three clusters; (b) three seed points selected as cluster centers and initial assignment of the data points to clusters; (c) and (d) intermediate iterations updating cluster labels and their centers; (e) final clustering obtained by K-means algorithm at convergence

\section{FEATURE EXTRACTION}

The feature extraction is extracting the cluster which shows the defect in image at the K-mean output. The extracted cluster is given to the thresholding process. It applies binary mask over the entire image. It makes the dark pixel become darker and white become brighter. In threshold coding, each transform coefficient is compared with a threshold. If it is less than the threshold value then it is considered as zero. If it is larger than the threshold will be considered as one. The thresholding method is an adaptive method where only those coefficients whose magnitudes are above a threshold are retained within each block. Let us consider an image 'f that have the $\mathrm{k}$ gray level. An integer value of threshold $\mathrm{T}$, which lies in the gray scale range of $\mathrm{k}$. The thresholding process is a comparison. Each pixel in ' $\mathrm{f}$ ' is compared to T. Based on that, binary decision is made. That defines the value of the particular pixel in an output binary image ' $\mathrm{g}$ ':

$$
g(n)=\left\{\begin{array}{l}
{ }^{\prime} 0 ' \text { if } f(n) \geq T \\
{ }^{\prime} 1^{\prime} \text { if } f(n)<T
\end{array}\right.
$$

\section{APPROXIMATE REASONING}

In the approximate reasoning is calculate the defected area is using the binarization method.[8] That is the image having only two values either black or white ( 0 or 1$)$. Here $256 \times 256$ jpeg image is a maximum image size. The binary image can be represented as a summation of total number of white and black pixels. Therefore image (I) is,

$I=\sum_{w}^{255} \sum_{H=0}^{255}[f(0)+f(1)] \quad-(4)$

Pixels $=$ Width $(\mathrm{W}) \mathrm{X}$ Height $(\mathrm{H})=256$ X 256

$\mathrm{f}(0)=$ white pixel $($ digit 0$), \mathrm{f}(\mathrm{l})=$ black pixel (digit 1$)$.

Therefore, No. of white pixel(P)-

$P=\sum_{w}^{255} \sum_{H=0}^{255} f(0)$

Where,

$\mathrm{P}=$ number of white pixels (width*height), One Pixel $=0.264 \mathrm{~mm}$.

Therefore the defect area calculation formula is-

$S=[(\sqrt{P}) 0.264] \mathrm{mm}^{2} \quad-(6)$

$\mathrm{P}=$ no-of white pixels; $\mathrm{W}=$ width; $\mathrm{H}=$ height.

VIII.

CONCLUSION

In this paper, we have introduced the computer based new K-Mean algorithm for defect detection. This algorithm is based upon clustering techniques. The basis of segmentation is used in this clustering algorithm to spot the defect in the fabric. The algorithm is developed in MATLAB for analysis and defect identification. KMean clustering produces fairly high accuracy i.e. $96 \%$ and requires fairly less computation time as compared other existing algorithms.

\section{REFERENCE}

[1] O. Miljkovi'c . Image Pre-processing Tool. Kragujevac J. Math. 32 (2009) 97-107.

[2] T. Cervinka, and I. Provazn'1k. Pre-processing for Segmentation of Computer Tomography Images. The Faculty of Electrical Engineering and Communication Brno University of Technology, Department of Biomedical Engineering, 2007.

[3] S. ANITHA, and V. RADHA. "Comparison of Image Preprocessing Techniques for Textile Texture Images". International Journal of Engineering Science and Technology, Vol. 2(12),2010, 7619-7625.

[4] Mark Grundland*, Neil A. Dodgson" Decolorize: fast, contrast enhancing, color to grayscale conversion"

[5] Raymond H. Chan, Chung-Wa Ho, and Mila Nikolova" Salt-and-Pepper Noise Removal by Median-Type Noise Detectors and Detail-Preserving Regularization". 
[6] Zhou Wang and David Zhang" Progressive Switching Median Filter for the Removal of Impulse Noise from Highly Corrupted Images

[7] Zhe LIU and Xiuchen WANG "Image Defect Recognition Based on "Super Fuzzy" Characteristic" JOURNAL OF MULTIMEDIA, VOL. 5, NO. 2, APRIL 2010.

[8] J.selvakumar, A.Lakshmi," Brain Tumor Segmentation and Its Area Calculation in Brain MR Images using K-Mean Clustering and Fuzzy C-Mean Algorithm" IEEE-International Conference On Advances In Engineering, Science And Management (ICAESM -2012) March 30, 31, 2012.

[9] Anil K. Jain "Data clustering: 50 years beyond K-means" Department of Computer Science and Engineering, Michigan State University, East Lansing, Michigan 48824, USA.

[10] Khaled Alsabti, Sanjay Ranka ,Vineet Singh” An Efficient K-Means Clustering Algorithm”

[11] Tapas Kanungo a, David M. Mount b,, 1, Nathan S. Netanyahu c,d, Christine D. Piatko e Ruth Silverman $\mathrm{d}$, Angela Y. Wu f' A local search approximation algorithm for $k$-means clustering.

[12] Rafael C.Gonzalez "Digital Image Processing using MATALB".

[13] www.wikipedia.com 